\title{
Hypotensive Effect of FAD and Its Mechanism: Involvement of Nitric Oxide Synthase Activation
}

\author{
Akiko Hashida-OKumura, ${ }^{1, *}$ Katsuya NagaI, ${ }^{1}$ Nobuaki OKumura, ${ }^{1}$ \\ Nobuo NAGAI, ${ }^{1}$ Hiroshi Hibino, ${ }^{1}$ Akira NiIJImA, ${ }^{2}$ \\ and Hachiro NAKAGAWA ${ }^{1}$ \\ ${ }^{1}$ Division of Protein Metabolism, Institute for Protein Research, Osaka University, \\ Suita 565, Japan \\ ${ }^{2}$ Department of Physiology, Niigata University, Niigata 951, Japan
}

(Received October 21, 1994)

\begin{abstract}
Summary Flavin adenine dinucleotide (FAD) is one of the cofactors of nitric oxide synthase (NOS). Recently, we found that FAD acted as an allosteric activator of holo NOS of the neuronal type. In the present study, we obtained the following results in rats: 1) FAD enhanced cyclic GMP (cGMP) production in primary cultures of neuronal cells and its effect was completely abolished by preincubation of the cells with a potent inhibitor of NOS, $\mathrm{N}^{\mathrm{G}}$-monomethyl-L-arginine. 2) FAD injection into the lateral cerebral ventricle decreased the blood pressure and heart rate. This effect was suppressed by preadministration of $\mathrm{N}^{\mathrm{G}}$-monomethylL-arginine. 3) The reduction in the blood pressure and heart rate by FAD was accompanied by suppression of the neural activity of the sympathetic efferents to the kidney. This effect was blocked by preadministration of $\mathrm{N}^{\mathrm{G}}$-monomethyl-L-arginine. 4) Intravenous injection of FAD also caused reductions in the blood pressure and heart rate. These results suggest that FAD activates NOS in neuronal cells and that the resultant NO reduces the blood pressure by suppressing sympathetic nerve activity. We also obtained evidence that FAD regulates the cardiovascular system when administered peripherally.
\end{abstract}

Key Words: cyclic GMP, blood pressure, sympathetic nervous system, nitric oxide, primary cultures of neuronal cells

Nitric oxide (NO) is involved in a variety of physiological events such as neurotransmission [1], vasodilation [2], and the cytotoxic action of the immune system [3] and is produced from L-arginine by nitric oxide synthase (NOS). There

*To whom correspondence should be addressed. 
are reports that its activity is regulated transcriptionally or posttranscriptionally, depending on cell type and isoform, and that its expression in macrophages [4], hepatocytes [5] and vascular smooth muscle cells [6] is induced by cytokines. On the other hand, in neuronal cells and endothelial cells NOS expression was found to be constitutive, its activity depending on $\mathrm{Ca}^{2+} /$ calmodulin. In vascular endothelial cells, NOS synthesizes NO in response to various agonists such as acetylcholine [7], ATP, and bradykinin [8] through increases in intracellular $\mathrm{Ca}^{2+}$ concentration [9]. In neuronal cells, NOS activity depends on NADPH, tetrahydrobiopterin, flavin mononucleotide (FMN), and flavin adenine dinucleotide (FAD), as well as on $\mathrm{Ca}^{2+} /$ calmodulin. Neuronal NOS contains tightly, but noncovalently, bound FAD [10,11].

Neuronal NOS is activated when cells are stimulated by glutamate. Glutamate is released from presynaptic terminals and acts on the N-methyl-D-aspartate (NMDA) receptor of the postsynaptic membrane, resulting in increased influx of $\mathrm{Ca}^{2+}$ and activation of NOS via the $\mathrm{Ca}^{2+} /$ calmodulin system $[12,13]$. The NO formed diffuses out from the postsynaptic membrane and activates soluble guanylate cyclase in the presynaptic terminals and astrocytic processes [13], resulting in an increase in intracellular cyclic GMP (cGMP). This suggests that NO might be involved in the regulation of the signal transduction system in the brain. Consistent with this idea, NO was shown to play a critical role in central regulation of sympathetic tone $[14,15]$.

Recently we found a factor in both rat urine and bovine adrenals that activated neuronal NOS and reduced the blood pressure [16]. We found that this factor resembled FAD in its profile of elution from an anion-exchange column (MonoQ column, Pharmacia, Uppsala), its yellow color, and its loss of activity on treatment with phosphodiesterase. Then we examined the effect of FAD on neuronal NOS. We found that FAD counteracted the inhibition by L-arginine by releasing the negative cooperativity on its activity besides acting as a cofactor (unpublished observation). Here we report the effect of FAD on NOS in primary cultures of neuronal cells in vitro and on the blood pressure in vivo. We also discuss the relationship between the two phenomena and their possible mechanism.

\section{MATERIALS AND METHODS}

Materials. FAD, FMN, riboflavin, calmodulin, poly-L-lysine, urethane, $\mathrm{N}^{\mathrm{G}}$ monomethyl-L-arginine (L-NMMA), and 2',5'-ADP agarose were purchased from Sigma Chemical Co., St. Louis, MO. (6R)-5,6,7,8-tetrahydrobiopterin was purchased from Research Biochemicals International, Natick, MA. Dulbecco's modified Eagle's medium (DMEM) was obtained from Nissui Pharmaceutical Co., Ltd., Tokyo; calf and horse sera were from HyClone Laboratories, Inc., Logan, UT. All other chemicals were from Wako Pure Chemical Industries, Osaka, unless otherwise specified. 
Animals. Male Wistar rats, weighing 250-300 g, were used. They were allowed free access to food (laboratory chow pellets, type MF, Oriental Yeast Co., Tokyo) and water, and were kept for at least 1 week before experiments in a room maintained at $24 \pm 1^{\circ} \mathrm{C}$ and illuminated for $12 \mathrm{~h}$ a day $(0700-1900 \mathrm{~h})$.

Partial purification of NOS from rat brain. Neuronal NOS was purified as described previously [17], with minor modifications. Briefly, whole rat brains were homogenized in buffer A [25 mM Tris- $\mathrm{HCl}(\mathrm{pH} 7.4), 0.5 \mathrm{~mm}$ EDTA, $1 \mathrm{~mm}$ dithiothreitol and leupeptin $(10 \mathrm{mg} / \mathrm{ml})]$ and centrifuged at $30,000 \times g$ for $30 \mathrm{~min}$. The supernatant was subjected to $2^{\prime}, 5^{\prime}$-ADP agarose affinity chromatography, and material was eluted with $10 \mathrm{mM}$ NADPH in buffer A. The eluate was applied to an FPLC MonoQ anion-exchange column and eluted with a gradient of $0-0.4 \mathrm{M}$ $\mathrm{NaCl}$ in buffer A. By this column chromatography, we obtained a single peak of NOS activity and used this fraction for examination of the effect of FAD.

NOS activity assay. NOS activity was determined by measuring the formation of citrulline. The complete reaction mixture $(300 \mu 1)$ contained a partially purified preparation of NOS (15 $\mu$ g protein), $50 \mathrm{mM}$ Tris- $\mathrm{HCl}(\mathrm{pH} 7.4), 1 \mathrm{~mm}$ NADPH, $1.3 \mathrm{mM}$ L-arginine, calmodulin $(6 \mu \mathrm{g} / \mathrm{ml}), 1.6 \mathrm{mM} \mathrm{CaCl}_{2}, 50 \mu \mathrm{M}(6 \mathrm{R})$ 5,6,7,8-tetrahydrobiopterin, and flavins. The mixture was incubated for $5 \mathrm{~min}$ at $37^{\circ} \mathrm{C}$ and then boiled for $30 \mathrm{~s}$ at $100^{\circ} \mathrm{C}$ to stop the reaction. The citrulline formed in the reaction mixture was then measured by the method of Schmidt et al. [18]. Briefly, $300 \mu 1$ of the sample was treated with urease $(100 \mathrm{mU})$ for $30 \mathrm{~min}$ at $37^{\circ} \mathrm{C}$. Then $600 \mu \mathrm{l}$ of acid solution $\left[\mathrm{H}_{2} \mathrm{SO}_{4}: \mathrm{H}_{3} \mathrm{PO}_{4}: \mathrm{H}_{2} \mathrm{O}=25: 20: 55(\mathrm{v} / \mathrm{v})\right.$ containing $\mathrm{FeCl}_{3} \cdot 6 \mathrm{H}_{2} \mathrm{O}(83.4 \mathrm{mg} /$ liter $\left.)\right]$ and $300 \mu \mathrm{l}$ of reagent solution $[0.01 \%(\mathrm{w} / \mathrm{v})$ thiosemicarbazide and $0.5 \%(\mathrm{w} / \mathrm{v})$ diacetylmonoxime] were added, and the mixture was boiled for $15 \mathrm{~min}$ and centrifuged for $5 \mathrm{~min}$ at $5,000 \times \mathrm{g}$. The absorption of the supernatant was then measured at $540 \mathrm{~nm}$.

Neuronal cell culture. Primary cultures of neuronal cells were prepared from the brains of fetal rats at 18 days of gestation as described previously [19]. The cells were suspended at $2 \times 10^{6}$ cells per $\mathrm{ml}$ in DMEM containing $5 \%$ calf serum and $5 \%$ horse serum. Cells in a volume of $2 \mathrm{ml}$ were inoculated into $35-\mathrm{mm}$ tissue culture dishes coated with poly-L-lysine. Two days later, cytosine arabinoside was added at a final concentration of $10 \mu \mathrm{M}$. The medium was replaced by DMEM containing 5\% calf serum and 5\% horse serum without cytosine arabinoside 2 days thereafter. Five hours before cGMP measurement, the medium was replaced by DMEM without sera. When indicated, L-NMMA was added $2 \mathrm{~h}$ before the assay at a final concentration of $5 \mathrm{mM}$. Cyclic GMP production was measured within 7 days after preparation of cells.

Determination of cGMP production. 3-Isobutyl-1-methylxanthine was added to neuronal cells at a final concentration of $0.5 \mathrm{~mm} 1 \mathrm{~min}$ before stimulation. The cells were stimulated with various concentrations of FAD or glutamate in a volume of $100 \mu \mathrm{l}$ (final concentration, $200 \mu \mathrm{M}$ ) and incubated for $5 \mathrm{~min}$ at $37^{\circ} \mathrm{C}$. Then they were washed 3 times with ice-cold phosphate-buffered saline (PBS) and collected in $400 \mu 1$ of PBS. The suspension was mixed with ice-cold 
ethanol at a final concentration of $65 \%$, and centrifuged for $5 \mathrm{~min}$ at $5,000 \times \mathrm{g}$. The supernatant was dried under vacuum, and its cGMP concentration was determined by radioimmunoassay with a Yamasa cGMP assay kit (Yamasa Co., Tokyo).

Measurement of intracellular $\mathrm{Ca}^{2+}$ concentration. Neuronal cells were cultured on a cover glass with a Flexiperm chamber (Heraeus Co., Germany). The cells were loaded with $5 \mu \mathrm{M}$ Rhod 2-AM (Dojindo Co., Kumamoto) in buffer B (Krebs-Ringer phosphate buffer containing $5 \mathrm{mM}$ glucose and $500 \mu \mathrm{M} \mathrm{L}$-arginine). After $30 \mathrm{~min}$, they were washed 3 times with buffer $\mathrm{B}$ and incubated in $0.5 \mathrm{ml}$ of buffer $\mathrm{B}$ for $30 \mathrm{~min}$. Then the intracellular $\mathrm{Ca}^{2+}$ concentration was measured by monitoring the fluorescence intensity by a digital image analyzing technique (ARGUS-200/Ca, Hamamatsu Photonics, Hamamatsu, Japan) as described by Habara and Kanno [20]. Rhod 2 fluorescent images (at $580 \mathrm{~nm}$ ) were obtained by excitation at $553 \mathrm{~nm}$.

Measurement of blood pressure. Rats were anesthetized with urethane $(1 \mathrm{~g} /$ $\mathrm{kg}$, i.p.), and a cannula of polyethylene tubing (PE-10, Clay Adams, NJ) was then inserted into the right lateral cerebral ventricle (LCV). Another catheter of polyethylene tubing (PE-50, Clay Adams, NJ) was inserted into the left femoral artery for measurement of the blood pressure. FAD, FMN, or artificial cerebrospinal fluid (aCSF) [21] was introduced into the LCV via the catheter, and the mean arterial blood pressure was recorded using an amplifier (Star Medical, Co., Tokyo) equipped with a transducer to which the other end of the arterial catheter was connected.

For examination of the effect of the intravenous injection on the blood pressure, FAD, FMN, or saline was injected through a catheter inserted into the right femoral vein instead of the LCV.

Measurement of neural activity of sympathetic efferents to the kidney. The neural activity of the sympathetic efferents to the kidney was measured as described previously [22]. Rats weighing about $300 \mathrm{~g}$ were anesthetized with urethane, and an LCV cannula was inserted as described above together with a tracheal cannula. Under a dissecting microscope, nerve filaments of the sympathetic efferents to the kidney were isolated from the central cut end to record the neural activity with a pair of silver wire electrodes. Neural activity was amplified, displayed on an oscilloscope, and stored on digital audio tape. The neural activity was analyzed after conversion of the raw data to standard pulses with a window discriminator that separated discharges from background noise. A rate meter with a 5-s reset time was used to observe the time course of change in nerve activity, which was recorded with a pen recorder.

\section{RESULTS}

\section{Effects of flavins on NOS}

Figure 1 shows the effects of various flavin compounds on NOS activity of a partially purified preparation from rat brain. Consistent with our unpublished 
observations, FAD at $10 \mu \mathrm{M}$ activated NOS about twofold. FMN stimulated NOS activity in a similar manner to FAD, causing greatest stimulation at $10 \mu \mathrm{M}$. On the other hand, riboflavin inhibited NOS slightly. FAD, but not FMN, decreased the blood pressure when injected into the LCV and femoral vein, and increased the intracellular cGMP level as described below. Accordingly, only the effect of FAD and its mechanism were examined.

FAD-induced increase in cGMP content in primary cultures of neuronal cells

We examined whether extracellular administration of FAD affected NOS in neuronal cells by using primary cultures of neuronal cells prepared from fetal rat

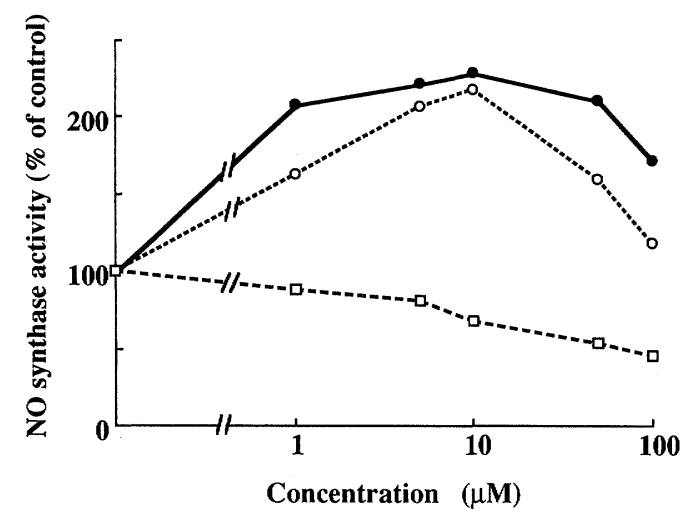

Fig. 1. Effects of FAD, FMN, and riboflavin on rat brain NOS. A preparation of NOS purified from rat brains by $2^{\prime}, 5^{\prime}$-ADP agarose and MonoQ column chromatography was used. Enzyme activities in the presence of various concentrations of FAD (closed circles), FMN (open circles), and riboflavin (open squares) were compared.

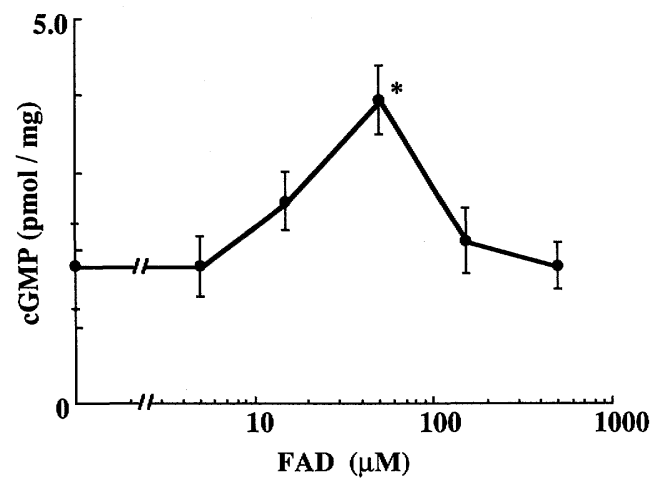

Fig. 2. Effect of FAD on cGMP production in primary cultures of neuronal cells. 3Isobutyl-1-methylxanthine (final concentration, $0.5 \mathrm{~mm}$ ) was added to primary cultures of neuronal cells $1 \mathrm{~min}$ before stimulation. Then cells were stimulated with various concentrations of FAD for $5 \mathrm{~min}$. Values are means $\pm S E M$ of triplicate determinations. ${ }^{*} p<0.05$ indicates a significant difference from the control by the $t$-test.

Vol. 18, No. 2, 1995 
brain. As NO is known to stimulate cytoplasmic guanylate cyclase [1], the intracellular cGMP level was measured after incubation of the cells with FAD to determine the effect of FAD on NOS activity. As shown in Figs. 2 and 3, cGMP production in primary cultures of neuronal cells was markedly and significantly increased by FAD at $50 \mu \mathrm{M}$, its level being comparable to that observed with glutamate $(200 \mu \mathrm{M})$, a physiological agonist for NOS in neuronal cells (Fig. 3). FMN did not affect the intracellular cGMP level (data not shown). Moreover, the finding that FAD-induced cGMP production was completely blocked by the addition of L-NMMA, an inhibitor of NOS, supports the conclusion that cGMP accumulation induced by FAD resulted from activation of neuronal NOS (Fig. 3).

\section{Effect of $F A D$ on the intracellular $\mathrm{Ca}^{2+}$ concentration}

Based on the proposition that in neuronal cells, activation of NOS is due to elevation of intracellular $\mathrm{Ca}^{2+}$ concentration, we examined whether the effect of FAD on cGMP production was mediated by $\mathrm{Ca}^{2+}$. For this purpose we determined the change in $\mathrm{Ca}^{2+}$ concentration in neuronal cells after FAD addition by monitoring the fluorescence intensity of a $\mathrm{Ca}^{2+}$ indicator, Rhod 2, with a digital image analyzer. We used Rhod 2 because FAD has strong fluorescence at $520 \mathrm{~nm}$ (excitation wavelength, $375 \mathrm{~nm}$ ), which interferes with the signals generated by most $\mathrm{Ca}^{2+}$ indicators such as Quin 2, Fra 2 and Fluo 3. Rhod 2 is unique in generating a fluorescence signal at a wavelength of $580 \mathrm{~nm}$, which is not interfered with by the fluorescence of FAD. As a positive control, $\mathrm{KCl}$ (final concentration, $75 \mathrm{~mm}$ ) was added to the cells to depolarize the plasma membrane; and this treatment enhanced the fluorescence intensity of Rhod 2 (Fig. 4B). However, no

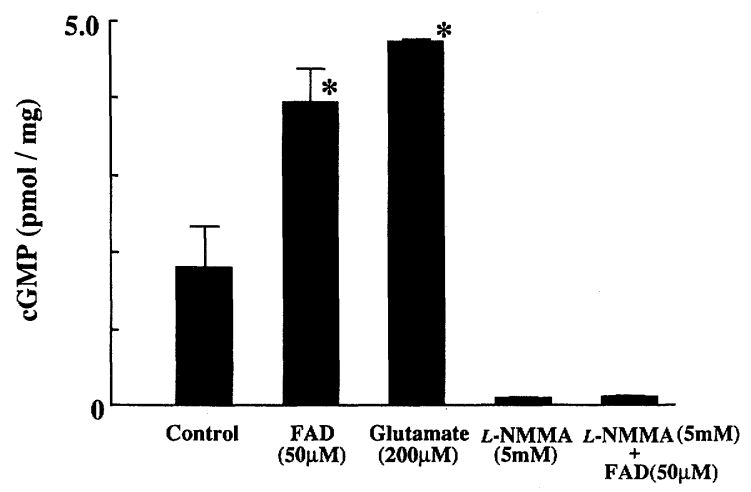

Fig. 3. Effects of various agents on cGMP production in primary cultures of neuronal cells. When indicated, L-NMMA (final concentration, $5 \mathrm{~mm}$ ) was added to primary cultures of neuronal cells $2 \mathrm{~h}$ before the experiment. Cells were treated with FAD, glutamate, or DMEM (control) in the presence of $0.5 \mathrm{mM}$ of 3-isobutyl-1-methylxanthine. Final concentrations are shown in parentheses under the names of reagents. Values are means \pm SEM for triplicate determinations. ${ }^{*} p<0.05$ indicates a significant difference from the control by the $t$-test. 
detectable change was observed in the intracellular $\mathrm{Ca}^{2+}$ concentration after the addition of FAD (final concentration, $50 \mu \mathrm{M}$ ) or Krebs-Ringer phosphate (control) (Fig. 4A and $\mathrm{C}$, respectively).

\section{Effects of flavin compounds on blood pressure and heart rate}

As both FAD and FMN activated rat brain NOS (Fig. 1), we examined their
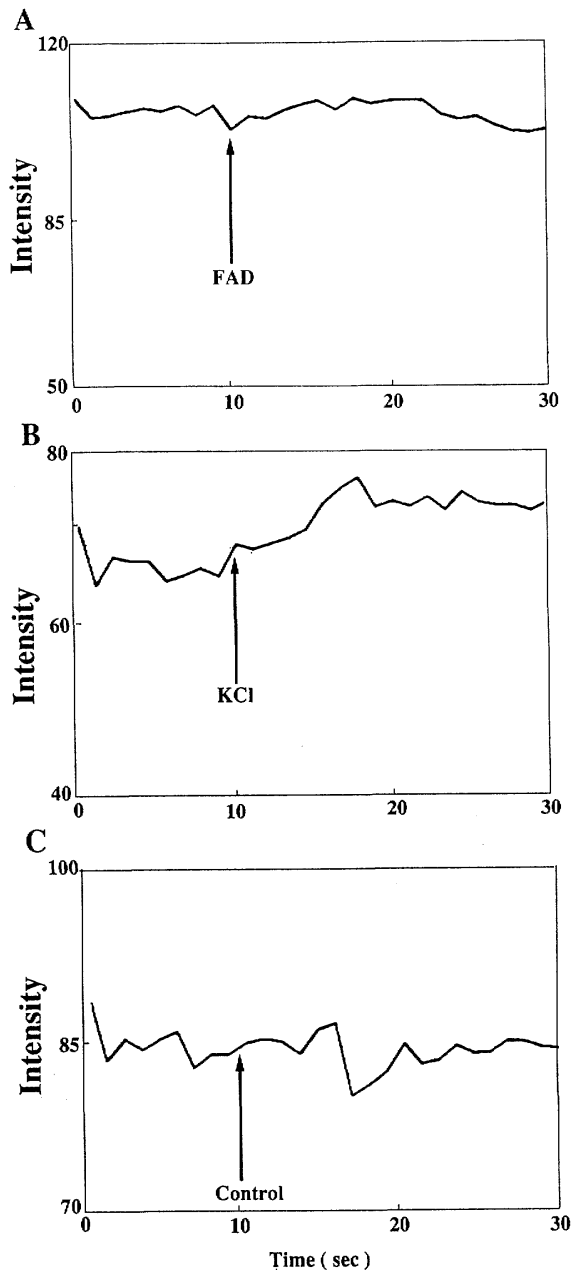

Fig. 4. Effect of FAD on the $\mathrm{Ca}^{2+}$ concentration in neuronal cells in primary culture. Neuronal cells were cultured on cover glasses with a Flexiperm as described in Materials and Methods. Volumes of $25 \mu 1$ of FAD (final concentration, $50 \mu \mathrm{M}$ ) (A), $\mathrm{KCl}$ (final concentration, $75 \mathrm{~mm}$ ) (B) and Krebs-Ringer phosphate (control, C) were added to the neuronal cell cultures at the points indicated by arrows. The intensity of the fluorescence of Rhod 2 was monitored by a digital image analyzing technique (Hamamatsu Photonics, Hamamatsu). The figure shows representative results from one of the five experiments performed.

Vol. 18, No. 2, 1995 
effects on the blood pressure. Injection of FAD into the LCV resulted in a decrease in the blood pressure (Fig. 5A). This hypotensive effect was associated with a reduction in the heart rate (Fig. $5 \mathrm{~B}$ ) and was blocked by preadministration of LNMMA (Fig. 6A, B). Intravenous injection of FAD also caused the reduction in the blood pressure (Fig. 5D) and heart rate (Fig. 5E). In contrast, neither LCV (Fig. 5C) nor intravenous injection of FMN (Fig. 5F) affected the blood pressure (or heart rate [not shown]). Neither aCSF injection into the LCV nor saline injection into the femoral vein as a control caused any change in the blood pressure and heart rate (data not shown). This experiment was repeated five times with similar results.

\section{Effect on sympathetic efferents to the kidney}

The fact that FAD markedly decreased the heart rate suggested that its
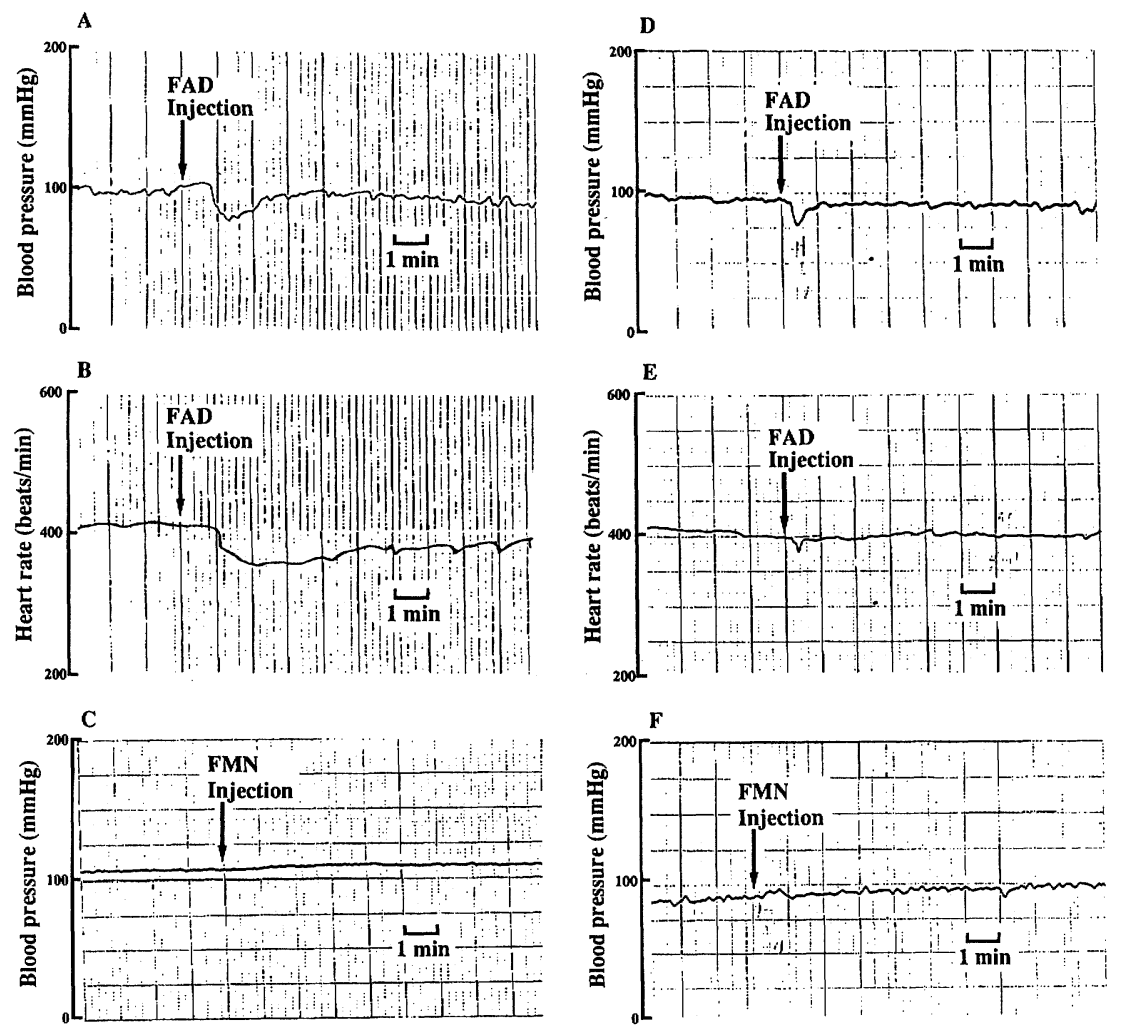

Fig. 5. Effects of FAD and FMN on the blood pressure and heart rate. FAD or FMN was dissolved in aCSF at a final concentration of $3 \mathrm{~mm}$ and injected in a volume of $10 \mu \mathrm{l}$ into the LCV of a rat anesthetized with urethane. Then the blood pressure $(A, C)$ and heart rate (B) were monitored. For peripheral administration, $200 \mu 1$ of $100 \mu \mathrm{M}$ FAD or FMN dissolved in saline was injected into the femoral vein of a rat anesthetized with urethane. Then the blood pressure $(D, F)$ and heart rate $(E)$ were measured. The result shown is a representative one from five experiments. 
hypotensive effect might be due to the decrease in the neural activity of the sympathetic nervous system. Therefore, we next examined whether FAD affected
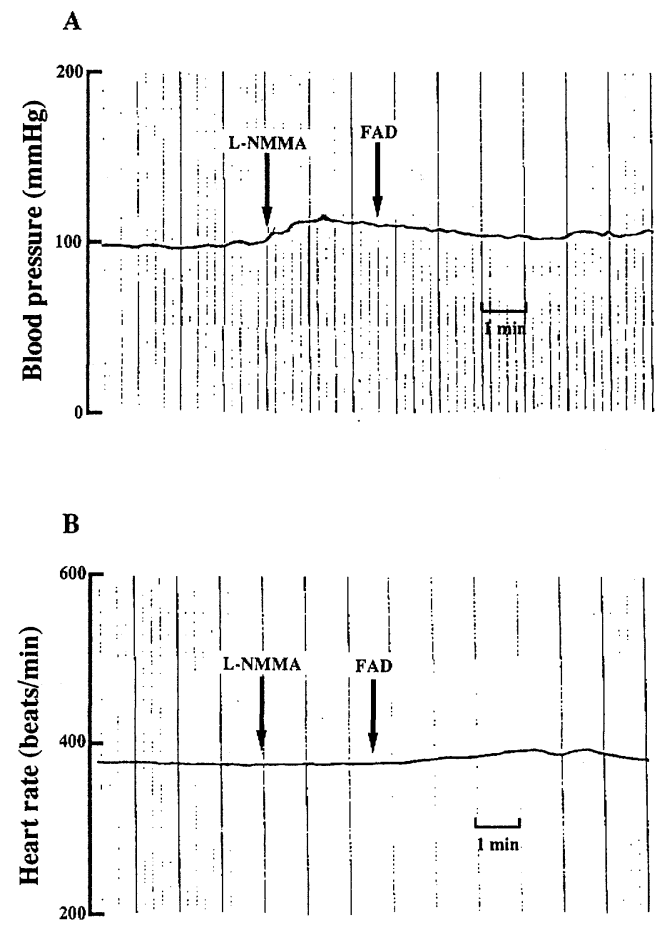

Fig. 6. Effect of preadministration of L-NMMA on the hypotensive effect by FAD. After $10 \mu 1$ of L-NMMA ( $2 \mu \mathrm{mol})$ had been injected into the LCV of a rat anesthetized with urethane, FAD $(3 \mathrm{~mm}, 10 \mu 1)$ was injected. Then the blood pressure (A) and heart rate (B) were monitored. The result shown is a representative one from five experiments.

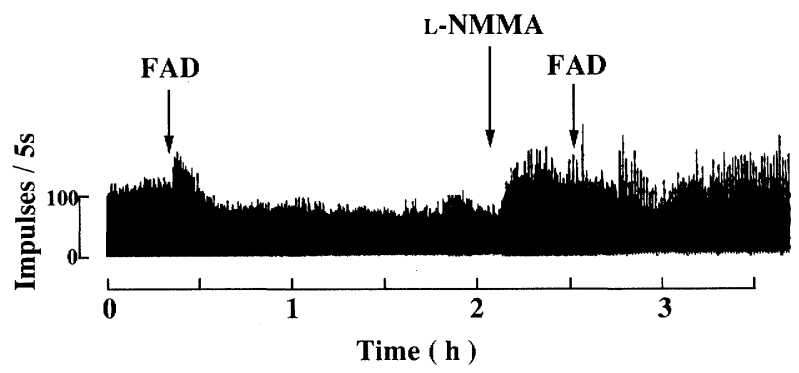

Fig. 7. Representative tracing depicting the effect of FAD on neural activity of sympathetic efferents to the kidney. First, FAD was dissolved in artificial CSF at a final concentration of $3 \mathrm{~mm}$ and injected in a volume of $10 \mu \mathrm{l}$ into the LCV of a rat anesthetized with urethane. Then, $10 \mu 1$ of L-NMMA ( $2 \mu \mathrm{mol})$ was injected into the LCV 20 min before the second FAD injection $(3 \mathrm{~mm}, 10 \mu \mathrm{l})$. The electrical activity of the sympathetic efferents to the kidney was monitored.

Vol. 18, No. 2, 1995 
the neural activity of the sympathetic efferents to the kidney. The sympathetic efferents innervating a unilateral kidney were isolated, and their electrical activity was recorded with a pair of electrodes. As shown in Fig. 7, injection of FAD into the LCV caused a temporary increase followed by a prolonged (more than $1.5 \mathrm{~h}$ ) suppression of the neural activity of the sympathetic efferents to the kidney. One hundred minutes after the first FAD injection, L-NMMA was administered into the LCV, and an increase in the neural activity of the sympathetic efferents was observed, as reported earlier by Sakuma et al. [14]. In such a condition, the second FAD injection caused only a temporary and slight suppression of the neural activity, but the neural activity returned to the elevated level soon. Artificial CSF injection into the LCV as a control did not cause any change in the neural activity of the sympathetic efferents to the kidney except for a temporary increase immediately upon the injection (data not shown). This temporary increase in the neural activity upon injection may be also caused via NO because it was not observed after administration of L-NMMA.

\section{DISCUSSION}

The present study indicated that exogenously administered FAD stimulated neuronal cells to induce a variety of responses including hypotension, bradycardia, and a decrease in sympathetic nervous activity.

FAD is a prosthetic group involved in electron-transfer reactions catalyzed by various oxidoreductases. Moreover, we obtained evidence that FAD was not only a prosthetic group, but also an allosteric effector of neuronal NOS (unpublished observation). NOS showed negative cooperativity in the presence of L-arginine at the concentrations more than $15 \mu \mathrm{M}$, which is much lower than that in serum (100 $\mu \mathrm{M})$. FAD activated NOS in a dose-dependent manner by counteracting the negative cooperativity, its effect reaching maximum at $10 \mu \mathrm{M}$. This concentration is much higher than that in serum ( $25 \mathrm{nM}$ ) or in brain cells (less than $3.5 \mu \mathrm{M}$ ). In view of these findings, it is possible that FAD functions as an activator of NOS in vivo.

The action of NO has been shown to be mediated, at least in part, by cGMP, whose level is regulated by cytoplasmic guanylate cyclase. In the present study, using primary cultures of neuronal cells, we found that FAD stimulated cGMP production and that cGMP production was almost completely blocked by pretreatment with L-NMMA, a potent inhibitor of NOS. These observations indicate that stimulation of the cells with FAD induced activation of neuronal NOS. Consistent with this conclusion, we recently detected FAD-induced NO production by using an NO-sensitive electrode (Intermedical Co., Tokyo) to measure directly its concentration in the medium of neuronal cell cultures (unpublished observation).

It is unknown how extracellular FAD stimulates NOS. It is thought that FAD is taken up into a cell after it has been hydrolyzed to riboflavin and phosphorylat- 
ed to it by an enzyme on the plasma membrane in the small intestine and liver. FAD may cross the plasma membrane by a similar mechanism as in the small intestine and liver, by other transport systems, or by simple diffusion to activate neuronal NOS directly in neuronal cells. The FAD concentration required for cell stimulation was higher than that for direct allosteric activation of NOS (maximal activation, $50 \mu \mathrm{M}$ for cell stimulation and $10 \mu \mathrm{M}$ for NOS activation). This difference can be explained by supposing that the efficiency of FAD transport into the cells was low. Another explanation is that FAD interacts with a cell surface receptor molecule to generate intracellular signals leading to stimulation of NOS. Neuronal NOS is a $\mathrm{Ca}^{2+} /$ calmodulin-dependent enzyme and is activated by agonists such as glutamate that induce calcium influx into the cells $[12,13]$. In the present study, however, FAD did not elicit any detectable change in intracellular $\mathrm{Ca}^{2+}$ concentration, judging from the fluorescence of the $\mathrm{Ca}^{2+}$ indicator Rhod 2 (Fig. 4). Thus the activation of NOS by FAD seems to be independent of any change in the $\mathrm{Ca}^{2+}$ concentration. NOS activity has also been suggested to be regulated by phosphorylation of the serine/threonine residues of the enzyme [11], although the physiological significance of this phosphorylation remains uncertain. NOS has a site for phosphorylation by cAMP-dependent protein kinase. Therefore, FAD may stimulate NOS activity by affecting a protein kinase. Further studies are needed to elucidate details of the activation mechanism of NOS.

In addition to FAD, FMN stimulated neuronal NOS and counteracted the inhibition of the enzyme by L-arginine in vitro, as the Hill coefficient was 1.0 in the presence of FMN (unpublished data). However, FMN did not affect the intracellular cGMP level when applied to primary cultures of neuronal cells or the blood pressure when injected into the LCV or femoral vein. Possibly, the efficiency of FMN transport is much lower than that of FAD or FMN has no receptor molecule with which to interact. It should be determined whether the effect of FAD in vivo is based on a different mechanism from that operating in vitro.

LCV injection of FAD decreased the blood pressure as well as the heart rate, and preadministration of L-NMMA blocked this effect. From these results, it is possible that FAD affected the activity of neuronal cells, especially neuronal cells of the autonomic nervous system via NO. This idea is supported by the findings that LCV administration of FAD reduced the electrical activity of the sympathetic nerves projected to the kidney and that preadministration of L-NMMA blocked this effect. L-NMMA, an NOS inhibitor, was found to increase the activity of the renal sympathetic nerve by inhibiting NOS in the brain [4]. Our findings are consistent with the idea that NO is involved in central regulation of the autonomic nervous system.

In vascular endothelial cells, NO is produced in response to various vasodilators and transmits a signal to smooth muscle cells. We found that intravenous injection of FAD caused a decrease in the blood pressure (Fig. 5). Possibly FAD causes vasodilation through activation of endothelial NOS by a direct action or by an interaction with some cell surface molecules such as adenosine receptors.

Vol. 18, No. 2, 1995 
The present results indicate that FAD may affect the cardiovascular system, probably by activating NOS in neurons and/or endothelial cells.

\section{REFERENCES}

1. Bredt, D.S., and Snyder, S.H. (1989): Nitric oxide mediates glutamate-linked enhancement of cGMP levels in the cerebellum. Proc. Natl. Acad. Sci. U.S.A., 86, 9030-9033.

2. Palmer, R.M.J., Ferrige, A.G., and Moncada, S. (1987): Nitric oxide release accounts for the biological activity of endothelium-derived relaxing factor. Nature, 327, 524-526.

3. Mills, C.D. (1991): Molecular basis of "suppressor" macrophages. Arginine metabolism via the nitric oxide synthetase pathway. J. Immunol., 146, 2719-2723.

4. Yui, Y., Hattori, R., Kosuga, K., Eizawa, H., Hiki, K., and Kawai, C. (1991): Purification of nitric oxide synthase from rat macrophages. J. Biol. Chem., 266, 12544-12547.

5. Evans, T., Carpenter, A., and Cohen, J. (1992): Purification of a distinctive form of endotoxin-induced nitric oxide synthase from rat liver. Proc. Natl. Acad. Sci. U.S.A., 89, 5361-5365.

6. Nunokawa, Y., Ishida, N., and Tanaka, S. (1993): Cloning of inducible nitric oxide synthase in rat vascular smooth muscle cells. Biochem. Biophys. Res. Commun., 191, 89-94.

7. Furchgott, R.F., and Zawadzki, J.V. (1980): The obligatory role of endothelial cells in the relaxation of arterial smooth muscle by acetylcholine. Nature, 288, 373-376.

8. Bogle, R.G., Coade, S.B., Moncada, S., Pearson, J.D., and Mann, G.E. (1991): Bradykinin and ATP stimulate L-arginine uptake and nitric oxide release in vascular endothelial cells. Biochem. Biophys. Res. Commun., 180, 926-932.

9. Hirata, Y., Emori, T., Eguchi, S., Kanno, K., Imai, T., Ohta, K., and Marumo, F. (1993): Endothelin receptor subtype $\mathrm{B}$ mediates synthesis of nitric oxide by cultured bovine endothelial cells. J. Clin. Invest., 91, 1367-1373.

10. Mayer, B., John, M., Heinzel, B., Werner, E.R., Wachter, H., Schultz, G., and Böhme, E. (1991): Brain nitric oxide synthase is a biopterin- and flavin-containing multi-functional oxido-reductase. FEBS Lett., 288, 187-191.

11. Bredt, D.S., Ferris, C.D., and Snyder, S.H. (1992): Nitric oxide synthase regulatory sites. $J$. Biol. Chem., 267, 10976-10981.

12. Knowles, R.G., and Moncada, S. (1994): Nitric oxide synthases in mammals. Biochem. J., 298, 249-258.

13. Garthwaite, J. (1991): Glutamate, nitric oxide and cell-cell signaling in the nervous system. Trends Neurosci., 14, 60-67.

14. Sakuma, I., Togashi, H., Yoshioka, M., Saito, H., Yanagida, M., Tamura, M., Kobayashi, T., Yasuda, H., Gross, S.S., and Levi, R. (1992): $\mathrm{N}^{\mathrm{G}}$-methyl-L-arginine, an inhibitor of Larginine-derived nitric oxide synthesis, stimulates renal sympathetic nerve activity in vivo. Circ. Res., 70, 607-611.

15. Harada, S., Tokunaga, S., Momohara, M., Masaki, H., Tagawa, T., Imaizumi, T., and Takeshita, A. (1993): Inhibition of nitric oxide formation in the nucleus tractus solitarius increases renal sympathetic nerve activity in rabbits. Circ. Res., 72, 511-516.

16. Hashida-Okumura, A., Nagai, K., and Nakagawa, H. (1994): Purification and characterization of nitric oxide synthase-activating factor. J. Clin. Biochem. Nutr., 17, 269-279.

17. Pollock, J.S., Förstermann, U., Mitchell, J.A., Warner, J.D., Schmidt, H.H.H.W., Banade, M., and Murad, F. (1991): Purification and characterization of particulate endotheliumderived relaxing factor synthase from cultured and native bovine aortic endothelial cells. Proc. Natl. Acad. Sci. U.S.A., 88, 10480-10484.

18. Schmidt, H.H.H.W., Wilde, P., Evers, B., and Böhme, E. (1989): Enzymatic formation of nitrogen oxides from L-arginine in bovine brain cytosol. Biochem. Biophys. Res. Commun., 165, 284-291. 
19. Hara, M., Matsuda, Y., Hirai, K., Okumura, N., and Nakagawa, H. (1989): Characteristics of glucose transport in neuronal cells and astrocytes from rat brain in primary culture. $J$. Neurochem., 52, 902-912.

20. Habara, Y., and Kanno, T. (1993): Dual effects of chlorobutanol on secretory response and intracellular $\mathrm{Ca}^{2+}$ dynamics in isolated pancreatic acini of the rat. Br. J. Pharmacol., 109, 685-692.

21. Sadashima, S., Thames, M., and Heistad, D. (1981): Cerebral blood flow during elevation of intracranial pressure: Role of sympathetic nerves. Am. J. Physiol., 241, H78-H84.

22. Niijima, A., Nagai, K., Nagai, N., and Nakagawa, H. (1992): Light enhances sympathetic and suppresses vagal outflows and lesions including the suprachiasmatic nucleus eliminate these changes in rats. J. Auton. Nerv. Syst., 40, 155-159. 